\title{
A Pharmacodynamic Strategy to Optimize Empirical Antibiotic Therapy for Gram-Negative Bacteria in a Brazilian Intensive Care Unit
}

\author{
Carlos R.V. Kiffer ${ }^{1}$, Joseph L. Kuti ${ }^{2}$, Caio M.F. Mendes ${ }^{1}$, Carmen P. Oplustil ${ }^{1}$, Jorge B. Amarante ${ }^{3}$, \\ Maria L. Biancalana ${ }^{3}$, Nelson Xavier ${ }^{3}$ and David P. Nicolau ${ }^{2}$ \\ ${ }^{1}$ Fleury Institute, São Paulo, SP; ${ }^{2}$ Center for Anti-infective Research and Development, Hartford Hospital, Hartford, Connecticut, USA; ${ }^{3}$ Service \\ of Infectious Diseases Control, Hospital Samaritano; São Paulo, SP, Brazil
}

\begin{abstract}
Pharmacodynamic analyses were proposed to determine optimal empirical antibiotic therapy against Gram-negative bacteria isolated in a Brazilian ICU. Due to high resistance rates, standard regimens of cefepime, ciprofloxacin, meropenem, and piperacillin/tazobactam were not able to attain significant bactericidal CFR. Prolonged infusion of meropenem achieved $88 \%$ CFR, making it a possible empirical regimen in this ICU until susceptibilities become available. Still, even through administration of high dose prolonged infusions, $\mathbf{1 2 . 0 \%}$ of simulated subjects did not achieve bactericidal exposure, suggesting that combination therapy would frequently be required in this setting. In conclusion, we recommend that in the presence of identified resistance problems among Gram-negative bacteria in a unit or hospital, MIC testing of formulary agents should be conducted along with pharmacodynamic simulation to assist in choosing an optimal antibiotic and dosage regimen for empirical use of severe infections until cultures and susceptibilities become available.
\end{abstract}

Key-Words: Pharmacodynamics, resistance, carbapenem, meropenem.

Inadequate antimicrobial therapy for severe infections is associated with increased mortality, prolonged stay in the intensive care unit (ICU), and increased risk of septic shock and bacteremia [1,2]. In most studies, the definition of inadequate antimicrobial therapy is based solely on a lack of in vitro susceptibility of the causative pathogen to the antibiotic administered. As a result, the use of a local antibiogram to determine empirical drug regimen combinations that would provide the greatest likelihood of at least one microbiologically active agent seems practical [3-5]. However, the simple classification of S, I, or R provides little information on the underlying level of susceptibility or resistance (i.e., the minimum inhibitory concentration [MIC]) [6]. This methodology also precludes a clear appreciation of the optimal dosage to administer, as it may be possible to overcome low levels of resistance in some patients with dosage enhancements [7]. As an alternative, we propose the determination of unit (or hospital) specific MIC distributions and use of pharmacodynamic modeling via Monte Carlo simulation to provide the best estimate of achieving optimal empirical antibiotic therapy.

In view of these facts and increasing resistance rates in Brazil, we set out to investigate which Gram-negative bacteria caused infections in an adult ICU of a tertiary care medical center (Hospital Samaritano) in São Paulo, Brazil and to determine the MIC distributions of several antibiotic therapies so that an optimal antibiotic and dosage regimen could be chosen empirically.

Received on 12 September 2006; revised 15 December 2006. Address for correspondence: Dr. Carlos R.V. Kiffer. Fleury Institute Setor de Microbiologia. Avenida General Waldomiro de Lima 508. Zip code: 04344-070 - Jabaquara - São Paulo - SP, Brazil. Phone: +55 11 50147657. Fax: +55 11 50147601. E-mail: carlos.kiffer@fleury.com.br.

The Brazilian Journal of Infectious Diseases 2007;11(2):183-185. (C) 2007 by The Brazilian Journal of Infectious Diseases and Contexto Publishing. All rights reserved.
Over a 9-month interval in 2004, Gram-negative bacteria $(n=43)$ were collected consecutively from adults treated for documented infections in the ICU of the referred hospital. During this period all possibilities of nosocomial outbreaks were ruled out by investigators and were validated externally by the hospital infection control service. Bacteria were isolated from the following infection sources: blood/catheter, $n=22$ (51\%); urine, $n=10$ (23\%), bronchoalveolar lavage, $n=8(19 \%)$, and abdominal/wound, $n=3$ (7\%). MIC testing was performed by E-test ${ }^{\circledR}$ methodology (ABBiodisk, Solna, Sweden). Reported MICs that fell within intervals for standard broth microdilution values reassigned the value of the next highest tube dilution based on these intervals. Pseudomonas aeruginosa was the most commonly isolated Gram-negative pathogen (Table 1). For all 43 Gram-negatives isolated, susceptibility rates were greatest for meropenem (81.4\%), followed by piperacillin/tazobactam (53.5\%), cefepime (48.9\%), and ciprofloxacin (39.5\%). The range of MICs isolated is also listed in Table 1.

Pharmacodynamic analyses were conducted via a 5000 subject Monte Carlo simulation (Crystal Ball 2000 v.2.2, Decisioneering Inc., Denver, CO, USA) for standard intravenous dosage regimens of cefepime, ciprofloxacin, meropenem, and piperacillin/tazobactam (dosage and antimicrobial drugs routinely used in the unit). All standard dosage regimens were simulated as 30-minute infusions via a 1-compartment intravenous infusion model. Estimates of pharmacokinetic parameters and dispersion were derived from studies in healthy volunteers as reported in past OPTAMA studies $[8,9]$. The bactericidal cumulative fraction of response (CFR) was calculated for each drug regimen against the bacteria population as a single group. Bactericidal CFR was defined as free drug concentrations above the MIC for a percentage of the dosing interval ( $\% f \mathrm{~T}>\mathrm{MIC}$ ) of $40 \%$ for meropenem, $50 \%$ for the other $\beta$-lactams and as a ratio of total drug area under the curve (AUC)/MIC greater than or equal 
Table 1. Frequency and antibiotic MIC range of 43 Gram-negative bacteria collected consecutively in the adult ICU of a Brazilian hospital over a 9 month period in 2004

\begin{tabular}{lccccc}
\hline & & \multicolumn{4}{c}{ MIC $(\mu \mathrm{g} / \mathbf{m L})$ range } \\
\cline { 3 - 6 } Bacteria & $\mathbf{N}(\%)$ & Cefepime & Ciprofloxacin & TZP & Meropenem \\
\hline P. aeruginosa & $15(35)$ & $2->256$ & $0.064->32$ & $4->256$ & $0.125->32$ \\
E. coli & $10(23)$ & $0.032-256$ & $0.006->32$ & $1.5-8$ & $0.012-0.047$ \\
E. cloacae & $6(14)$ & $0.094-192$ & $0.012->32$ & $4->256$ & $0.032-0.094$ \\
A. baumannii & $6(14)$ & $3->256$ & $0.125->32$ & $3->256$ & $0.25-16$ \\
Klebsiella spp. & $3(7)$ & $0.032-256$ & $0.012-6$ & $0.75-4$ & $0.023-0.064$ \\
Other & $3(7)$ & $0.125->256$ & $0.008-1.5$ & $0.75->256$ & $0.023->32$ \\
\hline
\end{tabular}

$\mathrm{TZP}=$ piperacillin/tazobactam.

Table 2. Cumulative fractions of response (CFR) at varied $\% f \mathrm{~T}>\mathrm{MIC}$ exposures for frequently used $\beta$-lactam regimens in a ICU in Brazil

\begin{tabular}{ccccc}
\hline \% f T >MIC & $\begin{array}{c}\text { Cefepime } \\
\text { 2g q12h }\end{array}$ & $\begin{array}{c}\text { Meropenem } \\
\text { 1g q8h }\end{array}$ & $\begin{array}{c}\text { TZP } \\
\mathbf{4 . 5 g ~ q 8 h}\end{array}$ & $\begin{array}{c}\text { TZP } \\
\mathbf{4 . 5 g ~ q 6 h}\end{array}$ \\
\hline 20 & 59.6 & 85.5 & 51.6 & 53.0 \\
30 & 54.0 & 83.5 & 49.3 & 50.8 \\
$40^{\mathrm{a}}$ & 48.1 & 80.7 & 46.9 & 49.2 \\
$50^{\mathrm{b}}$ & 47.2 & 73.2 & 37.0 & 47.5 \\
60 & 44.5 & 65.3 & 21.8 & 43.4 \\
70 & 41.4 & 60.3 & 10.7 & 33.0 \\
80 & 38.6 & 57.0 & 4.3 & 21.9 \\
90 & 35.6 & 54.0 & 1.1 & 13.0 \\
100 & 32.6 & 50.3 & 0.38 & 7.1 \\
\hline
\end{tabular}

$\mathrm{TZP}=$ piperacillin/tazobactam; all regimens admonistered as 30 minute infusions.

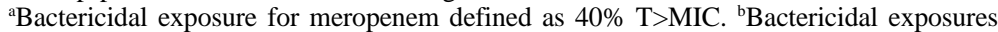
for the penicillins and cephalosporins defined as $50 \% f \mathrm{~T}>\mathrm{MIC}$.

to 125 for ciprofloxacin. CFR at alternative $\% f \mathrm{~T}>\mathrm{MIC}$ exposures were also calculated and reported. For those drug regimens with the highest bactericidal CFR, pharmacodynamically enhanced dosage strategies were explored to increase the CFR.

Bactericidal CFR was greatest for meropenem 1g every 8 hours (80.7\%), followed by piperacillin/tazobactam $4.5 \mathrm{~g}$ every 6 hours $(47.5 \%)$, cefepime 2 g every 12 hours (47.2\%), piperacillin/tazobactam 4.5 g every 8 hours (37.0\%), and ciprofloxacin 400 mg every 12 hours (34.9\%). CFR at alternative $\% f \mathrm{~T}>\mathrm{MIC}$ targets are listed in Table 2. While standard meropenem regimens provided the highest CFR and one similar to percent susceptibility, neither estimate should be considered optimal for empirical therapy. Thus, higher doses as prolonged infusions were simulated to improve CFR. A dose of $2 \mathrm{~g}$ administered as a 3-hour prolonged infusion every 8 hours achieved $88.0 \%$ likelihood of bactericidal CFR. Similar CFR probabilities were achieved up to $70 \% f \mathrm{~T}>$ MIC exposure with this regimen (i.e., $84.2 \%, 83.2 \%$, and $80.4 \%$ at $50 \%, 60 \%$, and $70 \% f \mathrm{~T}>\mathrm{MIC}$, respectively). Accordingly, this regimen would provide the greatest likelihood of optimal empirical therapy for patients in this ICU. Still, even through administration of high dose prolonged infusions, $12.0 \%$ of simulated subjects did not achieve bactericidal exposure, suggesting that combination therapy with an aminogylcoside would frequently be required in this setting.
The hospital antibiogram provides extremely valuable information for tracking emerging resistance throughout medical centers and has been proposed as useful tool for choosing empirical antibiotic therapy. However, in the presence of increasing resistance rates, the antibiogram provides little information on how to empirically dose antibiotics to improve the likelihood of optimal therapy and outcomes [6]. The currently proposed strategy for this ICU was designed to extend the interpretation of appropriate antibiotic therapy from simply in vitro susceptibility testing to a quantitative assessment of achievable in vivo antibiotic exposure. Although not routinely used, this approach is not novel; pharmacodynamic simulations in conjunction with MIC data have been used on several occasions to recommend specific dosage regimens on national, hospital and even individual patient levels [7, 10-14]. It was not under the scope of this analysis to collect outcome data; however, it was our intention that any beneficial regimen emerging from this simulation model could be implemented into the unit's daily practice to provide optimal empirical therapy and improve outcomes. We made these data available for the hospital's staff and started a discussion forum for implementing prescription changes.

In conclusion, we recommend that in the presence of identified resistance problems among Gram-negative bacteria 
in a unit or hospital, MIC testing of formulary agents should be conducted along with pharmacodynamic simulation to assist in choosing an optimal antibiotic and dosage regimen for empirical use against severe infections until cultures and susceptibilities become available.

\section{References}

1. Kollef M.H., Sherman G., Ward S., Fraser V.J. Inadequate antimicrobial treatment of infections: a risk factor for hospital mortality among critically ill patients. Chest 1999;115:462-74.

2. Kollef M.H. Inadequate antimicrobial treatment: an important determinant of outcome for hospitalized patients. Clin Infect Dis 2000;31:S131-8.

3. Ibrahim E.H., Sherman G., Ward S., et al. The influence of inadequate antimicrobial treatment of bloodstream infections on patient outcomes in the ICU setting. Chest 2000;118:146-55.

4. Rello J., Gallego M., Mariscal D., et al. The value of routine microbial investigation in ventilator-associated pneumonia. Am J Respir Crit Care Med 1997;156:196-200.

5. Trouillet J.L., Chastre J., Vuagnat A., et al. Ventilator-associated pneumonia caused by potentially drug-resistant bacteria. Am J Respir Crit Care Med 1998;157:531-539.

6. Gillespie E.L., Kuti J.L., Nicolau D.P. When “S” doesn't mean success: the importance of choice of antibiotic and dose on clinical and economic outcomes of severe infections. Conn Med 2005;69:203-10.

7. Kuti J.L., Moss K.M., Nicolau D.P., Knauft F.R. Empiric treatment of multidrug-resistant Burkholderia cepacia lung exacerbation in a patient with cystic fibrosis: Application of pharmacodynamic concepts to meropenem therapy. Pharmacother 2004;24:1641-5.
8. Maglio D., Kuti J.L., Nicolau D.P. Simulation of antibiotic pharmacodynamic exposure for the empiric treatment of nosocomial bloodstream infections: a report from the OPTAMA program. Clin Ther 2005;27:1032-42.

9. Sun H.K., Kuti J.L., Nicolau D.P. Pharmacodynamics of antimicrobials for the empiric treatment of nosocomial pneumonia: a report from the OPTAMA Program. Crit Care Med 2005;33:2222-7.

10. Ambrose P.G., Bhavnani S.M., Jones R.N. Pharmacokineticspharmacodynamics of cefepime and piperacillin-tazobactam against Escherichia coli and Klebsiella pneumoniae strains producing extended-spectrum b-lactamases: report from the ARREST Program. Antimicrob Agents Chemother 2003;47:1643-6.

11. Drusano G.L., Preston S.L., Hardalo C., et al. Use of preclinical data for selection of a Phase II/III dose for evernimicin and identification of a preclinical breakpoint. Antimicrob Agents Chemother 2001;45:13-22.

12. Kiffer C.R.V., Mendes C., Kuti J.L., Nicolau D.P. Pharmacodynamic comparisons of antimicrobials against nosocomial isolates of Escherichia coli, Klebsiella pneumoniae, Acinetobacter baumannii and Pseudomonas aeruginosa from the MYSTIC surveillance program: the OPTAMA Program, South America 2002. Diagn Microbiol Infect Dis 2004;49:109-16.

13. Kuti J.L., Nightingale C.H., Quintiliani R., Nicolau D.P. Pharmacodynamic profile of continuously infused piperacillin/ tazobactam against Pseudomonas aeruginosa using Monte Carlo analysis. Diagn Microbiol Infect Dis 2002;44:51-7.

14. Tam V.H., Louie A., Lomaestro B.M., Drusano G.L. Integration of population pharmacokinetics, a pharmacodynamic target, and microbiologic surveillance data to generate a rational empiric dosing strategy for cefepime against Pseudomonas aeruginosa. Pharmacother 2003;23:291-5. 